\title{
LAS NIIF Y SU IMPACTO EN EL SECTOR COMERCIAL DE ELECTRODOMÉSTICOS DEL CANTÓN MILAGRO
}

\section{IFRS AND ITS IMPACT ON THE COMMERCIAL SECTOR OF ELECTRICAL APPLIANCES IN CANTON DEL MILAGRO}

\author{
David Richard Pincay Sancan ${ }^{1}$ \\ Jazmin Elsa Sánchez Astudillo² \\ Lucy Lastenia Tasigchana Aguilar ${ }^{3}$ \\ Gonzalo Lenin Serrano Mantilla ${ }^{4}$
}

1. Máster en Tributación y Finanzas. Docente y Director en proyecto de Investigación de la Universidad Estatal de Milagro. Ex - Director Financiero de la UNEMI (Ecuador). E-mail: dav coco@hotmail.com

2. Máster en Administración y Dirección de Empresas. Docente de la Universidad de Guayaquil. Analista de Investigación y Directora en proyecto de Investigación de la Universidad Estatal de Milagro (Ecuador). E-mail: jazmín sanchez4@hotmail.com

3. Máster en Pymes. Docente de la Unidad Educativa 17 de Septiembre (Ecuador). Email: lucytasig@hotmail.com

4. Máster en Administración de Empresas, Docente Titular de la Universidad Estatal de Milagro, Gerente de Sucursal Milagro Banco de Guayaquil (Ecuador). E-mail:

gserrano123456@hotmail.com

Citación sugerida:

Pincay Sancan, D.R., Sánchez Astudillo, J.E., Tasigchana Aguilar, L.L y Serrano Mantilla, G.L. (2017). Las NIIF y su impacto en el sector comercial de electrodomésticos del Cantón Milagro. 3C Empresa, $\begin{array}{lllll}\text { investigación } y & \text { pensamiento crítico, 6(1), 63-76. DOI: }\end{array}$ <http://dx.doi.org/10.17993/3cemp.2017.060129.63-76/>. 


\section{RESUMEN}

Las Normas Internacionales de Información Financiera (NIIF) y las Normas Internacionales de Contabilidad (NIC), regulan el sector empresarial a nivel nacional e internacional con normativas contables de obligatoriedad desde el año 2008 en Ecuador, y son herramientas que permiten al sector productivo presentar, de forma correcta, sus Estados Financieros a los entes de control como es la Superintendencia de Compañías. El cantón Milagro perteneciente a la provincia del Guayas, es uno de los tres cantones más influyentes dentro del ámbito comercial, dado que su ubicación en la Zona cinco, permite que transiten las mercaderías de la sierra, oriente y sur del país, las PYME ubicadas en este sector y dedicados a la compra-venta de electrodomésticos han generado nuevas plazas de trabajo, pero ¿Estarán preparadas para la aplicación de las NIIF? A continuación, se presenta una investigación empírica para analizar la aplicación de las NIC y su cumplimiento en las PYME de comercialización de electrodomésticos en el Cantón Milagro, en la que se identifica una clasificación de 3 grupos diferenciados por sectores de empresa en comercio, servicio e industria. Además, se evidencia las NIIF y NIC como fuente de información competitiva para los Estados Financieros y actualización de conocimientos para los contadores y dueños de estas empresas.

\section{ABSTRACT}

The International Financial Reporting Standards (IFRS) and International Accounting Standards (IAS), regulate the business sector at national and international levels with accounting standards mandatory from 2008 in Ecuador, and is a tool that allows the productive sector present their financial statements correctly to control entities such as the Superintendency of Companies, the canton Milagro belonging to the province of Guayas is one of the most influential third cantons within the commercial area as its location in Zone five, allow transiting goods saw, orients and south of the country, SME located in this sector and dedicated to buying and selling appliance has generated new jobs, but you be prepared for the implementation of IFRS? Then an empirical research is conducted to analyze the application of IAS and compliance in SME marketing appliances in the Canton Milagro, which rated 3 groups differentiated by sector company in trade, service and industry is identified. In addition IFRS and IAS is evidenced as a source of competitive information for the Financial Statements and updating of knowledge for accountants and owners of these companies.

\section{PALABRAS CLAVE}

Comercio, contabilidad, NIIF, Estados Financieros; Pequeñas y Medianas Empresas.

\section{KEYWORDS}

Trade, accounting, IFRS, Financial Statements; Small and medium businesses. 


\section{INTRODUCCIÓN}

Los empresarios de las PYME en Ecuador apuntan a la automatización como un proceso de eficiencia, pero surge la pregunta de si esto funcionará por sí solo, y si no funciona "Cómo Hacerlo" (Gardey, Fernandez \& Madueño, 2009). El presidente del Instituto de Empresas Familiares del Ecuador - IEFE, afirmó que el $80 \%$ de las empresas micro empresariales del país son de tipo familiar y $40 \%$ logra pasar de segunda a tercera generación, patriarcado de abuelo a hijo, enseñanzas de habilidades o conocimiento, confección de producto, manera de comercializarlos, nacimientos de nuevos negocios, herencia que se transmite y se mejora en cada generación que involucra más la innovación y el desarrollo (Ontañeda, 2008).

De acuerdo a esa época las exigencias de administración se limitaban a una buena atención y a tener un buen producto para obtener éxito, de ahí que muchas empresas que antes fueron exitosas se quedaron en el camino (González, Lacoba \& Loureiro, 2015), y muy pocas pudieron adaptarse o mantenerse, y un número limitado pudo crecer para convertirse en una gran empresa (Marchán, 2009).

El aprendizaje y la práctica evolutiva de la administración presentan a las PYME dos grandes desafíos, que al no lograr controlar se vuelven amenazas para sobrevivir en un mercado competitivo (Medina, 2013). También la reinvención constante del negocio, una de las falencias que se presentan es la no aplicación de las NIIF, siendo obligatoria en Ecuador a partir del año 2008, por parte de la Superintendencia de Compañías, pero se ha vuelto compleja su aplicación por varios factores tanto económicos como de conocimientos (Tasigchana, 2015).

De este modo, se comprueba la importancia de las NIIF para las PYME en Ecuador (Consejo de Normas Internacionales de Contabilidad - 2009). La investigación fue direccionada al sector comercial de electrodomésticos, empresas y microempresas que por desconocimiento de las NIIF, sus estados financieros presentan información no razonable, falencias detectadas por la Superintendencia de Compañías y que perjudican en la toma de decisiones gerenciales (Superintendencia de Compañías, 2010). Esto no solo es una problemática del sector comercial, a nivel mundial y latinoamericano se viene tomando pautas para mejorar la aplicación de las NIIF, (Cano, 2010).

Se justifica esta investigación por la necesidad de mejorar la presentación de información financiera a los entes de control, lo que volvería a la industria y microempresas más competitivas en el manejo de información económica (Justiniano, 2016). Milagro es uno de los cantones principales y productivos de la provincia del Guayas, al estar asentada una industria comercial azucarera como es el Ingenio Valdez trajo consigo mujeres y hombres de todo el país y al generar recursos, apostaron al comercio, creando una gran zona comercial y formación de Pequeñas y Medianas Empresas sin cultura tributaria y menos con aplicación a NIC (Sánchez y Pincay, 2013), por lo que se pretende presentar estrategias para la difusión y capacitación a los microempresarios, a través de gremios profesionales, asociaciones de comerciantes, Cámara de Comercio (Justiniano, 2016). 
Así, en este estudio se plantea como objetivo general analizar la aplicación de las NIIF y NIC en las PYME dedicadas a la comercialización de electrodomésticos en el cantón Milagro (Tasigchana, 2015). En concreto y para alcanzar dicho objetivo general se plantean los siguientes objetivos específicos:

a) Determinar los factores de no aplicación de NIIF en las PYME del cantón Milagro.

b) Analizar las ventajas de aplicación de NIC y NIIF en este sector.

c) Clasificar las PYME del cantón Milagro dedicadas a la comercialización de electrodomésticos, a través de sus productos para identificar su aplicación de NIIF.

El documento se ha estructurado como sigue: en primer lugar se realiza un análisis del marco teórico en el que se introduce las Normas Internacionales de Información Financiera y Pequeñas y Medianas Empresas, PYME. A continuación, se presenta la investigación empírica realizada en las empresas comerciales de electrodomésticos asentadas en la cabecera cantonal Milagro, seguidamente la discusión de los resultados, y se finaliza con las conclusiones y propuestas de mejoras.

\section{MARCO TEÓRICO}

\subsection{NACIMIENTO Y EVOLUCIÓN DE LAS PYME EN ECUADOR}

El nacimiento de las Pequeñas y Medianas Empresas da el nombre a las siglas PYME (Stumpo, 2004), capitales pequeños y medianos que han surgido en los mercados y apoyado a las economías tanto locales como internacionales. La planificación estratégica es un factor que permite observar esto en dos estructuras (Rodríguez, 2000).

La primera estructura y original de las empresas en mención, organizaciones formadas legalmente (Casero, Mogollón \& Urbano, 2005), donde el producto dado termina en los salarios del personal como reconocimiento al esfuerzo del día a día de su trabajo para que se obtengan resultados económicos positivos para la empresa y sus dueños (Urbano, Casero y Mogollón, 2007). Estas empresas dinamizan la economía en el sector formal con capitales progresistas (Lozares, 2003).

La segunda estructura agrupa a la economía familiar (Velasco, Parra, y García, 2011). Su origen nace en casa, producto de la creatividad y experticia de los jefes de hogar, que van quedando de generación en generación, donde han aprendido a sobrevivir con capitales pequeños, propios (Rodrigo, 2006) y muchos de ellos a través de los créditos empresariales o gubernamentales (Fosado, 2007).

La política ecuatoriana no se ha quedado atrás, así, el presidente, el economista Rafael Correa Delgado incluye en la Constitución de la República del año 2008, el sistema económico social y solidario, donde el ser humano es reconocido como un sujeto y fin, palabras que se rescatan del artículo 283 (Asamblea Nacional, 2008). 
Dentro del estudio realizado en Ecuador por parte del Instituto Nacional de Estadística y Censos (INEC) en el año 2010, y estudios realizado en otras ciudades del país mencionado, reflejó en minoría a las medianas-grandes y grandes empresas (Tobar, 2013), y en gran mayoría, de acuerdo al en el Censo Económico de 2010, las micro empresas, las pequeñas empresas y las medianas empresas, esto nos da a denotar que en nuestro país el sector MIPYMES va liderando el mercado económico y lo que ha permitido direccionarnos en la investigación a esta problemática ya que debe relacionarse con las Normas Internacionales de Información Financiera en cumplimiento de las obligaciones que contrae un negocio formal.

El estudio realizado por Rodríguez (2014), muestra una recopilación de datos importantes para el desarrollo de esta investigación, dentro del cantón Milagro, así: Indica que el 24\% del Producto interno Bruto lo aportan las PYMES, y que las MIPYMES atraen a más de 17 000 empleados, y que dentro del comercio lo refleja el $32.36 \%$ y un $45.14 \%$ el sector de servicio y su diferencia lo maneja las industrias. El sector mencionado en líneas anteriores, está conformado por restaurantes, hoteles, gabinetes, reparaciones de electrodomésticos, donde la inversión pública juega un rol importante para el desarrollo de estas empresas y negocios.

De acuerdo al a información del Registro Oficial №444 de Abril 2011, en el que aprueba la Ley de Economía Popular y Solidaria, se crea un organismo de control como la Superintendencia para fortalecer las finanzas populares, programa desarrollado por el MYPRO - FONDEPYME.

En el año 2015, a través del Plan Estratégico de Desarrollo elaborado por el Gobierno Autónomo Descentralizado (GAD), se evidencia que en el cantón Milagro las actividades de comercio se mantienen en el liderato, luego las actividades agrícolas.

Figura 1. Categorías de actividades-año 2015

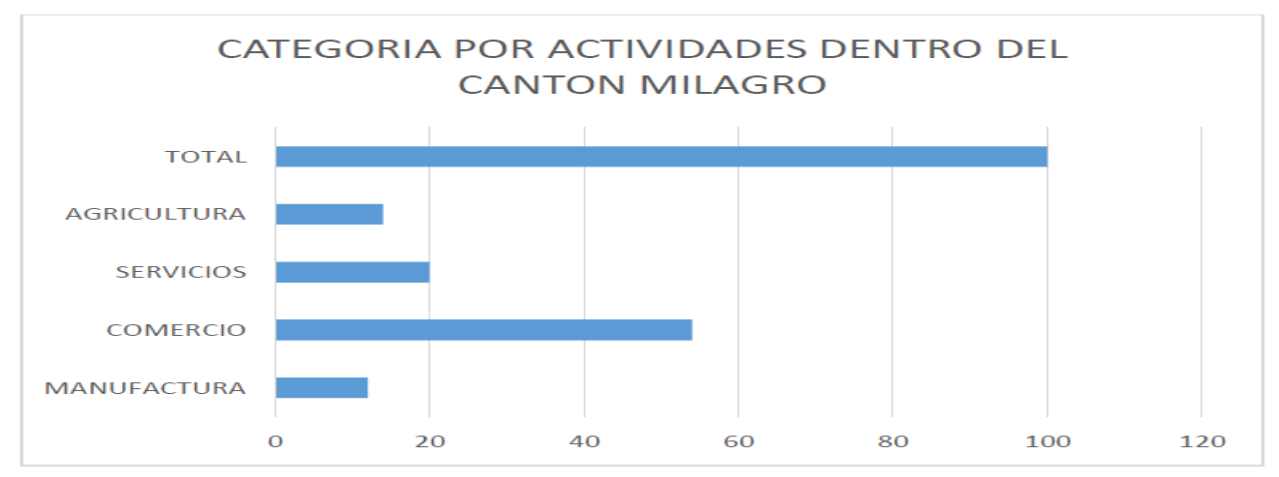

Fuente: Plan Estratégico de Desarrollo del GAD-Milagro 2015.

\subsection{LAS NIIF Y SU APLICACIÓN EN ECUADOR}

Las Normas Internacionales de Información Financiera, armonizan y mejoran la presentación de los estados financieros de las empresas a nivel mundial; así en Ecuador en el año 2008, mediante una resolución № 06 Q.ICl 004 del 21 de agosto del 2006, de la Superintendencia de Compañías, se adopta por obligación estas herramientas que 
cambiarían paulatinamente la aplicación de la normas contables, derogando las Normas Ecuatorianas de Contabilidad (NEC) por las Normas Internaciones de Contabilidad (NIC) (Sánchez y Pincay, 2013).

Para el año 2008 la Superintendencia de Compañías, mediante resolución №08.G.DSG.010, categorizó el uso de las normas disponiendo que su implementación se realizaría en 3 grupos, considerando años de transición y años de adopción (Superintendencia de Compañías, 2012)

Dentro del sector privado, refiriéndose al tercer grupo, están las Pequeñas y Medianas Empresas, PYME, que mediante el reordenamiento y clasificación de las empresas (Vásquez, 2014), empezaron con la aplicación de la normativa implementada por la Comunidad Andina de Naciones y la Superintendencia de Compañías (SC), en adopción de las NIIF (Superintendencia de Compañías, 2012). Para el año 2012 las PYME debieron cumplir con la transición de su información financiera y presentación, incluyendo las PYME de comercialización de electrodomésticos del cantón Milagro, provincia del Guayas.

Figura 2. Aplicación de Grupos en NIIF-2012.

\begin{tabular}{|c|lcc|}
\hline GRUPO & \multicolumn{4}{|c|}{ CONCEPTOS } & PERIODO \\
\hline 1 & $\begin{array}{l}\text { Compañías que cotizan en la bolsa de valores y } \\
\text { compañias de auditoria externa } \\
\text { Compañías con activos totales iguales o superiores a los } \\
4.000 .000,00 \text { al 31 de Diciembre del 2.007 } \\
\text { Compañías con activos totales menores a los } \\
4.000 \text { o00,00, valor bruto en ventas anuales inferior a } \\
5.000 .000,00 \text { y constan de menos de 200 trabajadores }\end{array}$ & 2012 PYMES \\
\hline
\end{tabular}

Fuente: Superintendencia de Compañías de Ecuador - 2012.

También hay que rescatar NIIF para PYME, creadas por Declaraciones de las Obligaciones de los Miembros (DOM 7) a través de las IASB Junta de Normas Internacionales de Contabilidad (IASB por su sigla en inglés). Cuando la NIIF para las PYME fue publicada en julio de 2009, el IASB asumió la tarea de evaluar la experiencia de las entidades en la aplicación de la NIIF para PYME, tras los primeros dos años de su adopción. Para ello, en junio de 2012, el IASB emitió una Solicitud de información: Revisión exhaustiva de la NIIF para las PYME y se incluyeron en las reformas del año 2013.

\section{METODOLOGÍA}

\subsection{ANÁLISIS EMPÍRICO}

La investigación empírica se inicia a través de una fase de naturaleza descriptiva cualitativa. En esta línea y siguiendo a García (2014), cualquier modificación supone un corte en el tiempo para analizar un momento concreto determinando, aspecto cualitativo, y obtener conclusiones, en la relación causa-efecto entre los estados financieros que emiten las PYME y la obligatoriedad de las NIIF. La investigación cualitativa se ha enfocado al desarrollo de las escalas aplicadas durante la entrevista y encuestas. Se presentaron a 
profesionales contables de las 30 empresas asentadas en el cantón Milagro dedicadas a la comercialización de electrodomésticos. Se mantuvieron entrevistas con 3 profesionales del sector productivo y 4 expertos del ámbito docente en NIIF, Emprendimiento y Contabilidad, fruto de estas entrevistas se eliminaron algunas preguntas y se cambió la redacción de otras, para mayor comprensión de los encuestados. El elemento muestral fue informante clave en cada una de las empresas seleccionadas, que sirvieron de nexo entre las PYME, su aplicación con las Normas Internacionales de Información Financiera, y sus contadores o dueños del negocios, 30 empresas fueron seleccionadas, dedicadas al comercio de electrodomésticos en el cantón Milagro. En la tabla 1 se presenta la ficha técnica del trabajo de campo llevado a cabo.

Tabla 1. Ficha técnica.

\begin{tabular}{|c|c|}
\hline Delimitación Geográfica & Ecuador \\
\hline Provincia & Guayas \\
\hline Cantón & Milagro \\
\hline Universo & Las PYME que comercializan electrodomésticos \\
\hline Muestreo & $\begin{array}{l}\text { Muestreo no probabilístico, se utilizó toda la población de } \\
\text { empresas que comercializan electrodomésticos }\end{array}$ \\
\hline $\begin{array}{l}\text { Instrumento de recolección de } \\
\text { datos }\end{array}$ & Cuestionario físico para encuesta y entrevistas \\
\hline Población & $\begin{array}{l}30 \text { PYME (Pequeñas y Medianas Empresas } \\
\text { comercializadoras de electrodomésticos) }\end{array}$ \\
\hline Fecha de realización & Junio - Octubre del 2015 \\
\hline Programa estadístico & SPSS versión 20.0 \\
\hline
\end{tabular}

Fuente: elaboración propia.- 2016.

\subsection{MEDICIÓN DE LA POBLACIÓN FINITA Y SUS VARIABLES}

El cuestionario se estructuró en partes conceptuales de aplicación a las normativas legales vigentes, como son las NIIF-NIC (IFRS, 2009), y la segunda parte en base al conocimiento y compromiso organizacional del personal a cargo de generar esta información. Sus variables responden a las hipótesis planteadas, la delimitación de la población parte de un estudio preliminar desarrollado en la UNEMI-2010 (Fernández, 2010), tuvo un total de 2066 de Pequeñas y Medianas Empresas, tal como se lo expone en la tabla 2, dividido por sectores de comercio, industria y servicio.

Tabla 2. Pequeñas y Medianas Empresas dentro del Cantón Milagro.

\begin{tabular}{|c|c|c|c|c|c|}
\hline \multirow{2}{*}{$\begin{array}{l}\text { CLASE DE } \\
\text { EMPRESA }\end{array}$} & \multicolumn{4}{|c|}{ TIPO DE EMPRESA } & \multirow[t]{2}{*}{ PORCENTAJE } \\
\hline & MEDIANA & MICRO & PEQUEÑA & TOTAL GENERAL & \\
\hline Comercial & 35 & 1115 & 127 & 1277 & $62 \%$ \\
\hline Servicios & 23 & 623 & 61 & 707 & $34 \%$ \\
\hline Industrial & 2 & 71 & 9 & 82 & $4 \%$ \\
\hline Total General & 60 & 1809 & 197 & 2066 & $100 \%$ \\
\hline
\end{tabular}

Fuente: Departamento de Investigación "Proyecto del Econ. Mario Fernández-2010 Unemi", (Fernández, 2010). 
Las empresas tomadas en consideración fueron treinta, consideradas de acuerdo a su línea de comercialización en electrodomésticos, se las visitó de forma personal, equipo de investigación, y se levantó el cuestionario de preguntas. Las empresas se describen en la tabla 3.

Tabla 3. Población encuestada (PYME).

\begin{tabular}{|c|c|c|}
\hline PERSONAS JURIDICAS & \multicolumn{2}{|c|}{ Personas naturales Obligados a Llevar Contabilidad } \\
\hline 1.Almacenes Piedra & 11. Comercial Pérez & 21. Comercial JOMI \\
\hline 2. Almacenes La Ganga & 12. Comercial Morán & 22. Créditos Shadai \\
\hline 3. Créditos Económicos & 13. Créditos J\&M & 23. Comercial Aida \\
\hline 4. Marcimex & 14. Comercial Pintag & 24. Comercial Alvarado \\
\hline 5. Artefacta & 15. Comercial Briana & 25. Créditos CHARLES \\
\hline 6. Almacenes Juan Eljuri & 16. Comercial Villacís & 26. Comercial López \\
\hline 7. Comandato & 17. Comercial Cabrera Molina & 27. Comercial David \\
\hline 8. Almacenes Jaher & 18. Créditos Milton & $\begin{array}{l}\text { 28.Comercial Gutiérrez } \\
\text { Cárdenas }\end{array}$ \\
\hline 9. Almacenes Japón & 19. Comercial Imperial & 29. Comercial Robles \\
\hline 10. Orve Hogar & 20. Créditos Romano & 30. Comercial Adrianita \\
\hline
\end{tabular}

Fuente: Empresas ubicadas en el Cantón Milagro.

Elaboración: Propia-2016.

\section{RESULTADOS}

\subsection{CARACTERÍSTICAS GENERALES DE LA MUESTRA}

Puede observarse en la tabla 3, de las 30 Pequeñas y Medianas Empresas que comercializan electrodomésticos dentro del Cantón Milagro, el 33\% tienen un registro único de contribuyente como persona jurídica, es decir además de rendir cuentas tributarias (Oria, 1997), deben contar con información de Estados Financieros ante la Superintendencia de Compañías, el $67 \%$ restante corresponde a locales que por sus niveles de venta y disposición tributaria están obligados a llevar contabilidad de acuerdo a las políticas y normas vigente, es decir cumpliendo con la adopción de las NIIF y NIC. El formato se encuentra preestablecido a niveles de cuentas de mayores, en el formulario de impuesto a la renta 101 y 102, emitido por el Servicio de Rentas Internas en su resolución NacDGERCGC15-0003211 y que se vincula con las bases de datos del ente de control de la información financiera del país "Superintendencia de Compañías", por la presentación del Estado de Situación Financiera y Estado de Resultados Integral. Por otra parte, se deduce que el $67 \%$ de informantes claves mantienen una informalidad en la información financiera, sin embargo se regula parcialmente por el cumplimiento tributario y permite ver la situación real financiera de este grupo de PYME. 
Gráfico 1. Estados Financieros conforme a NIIF

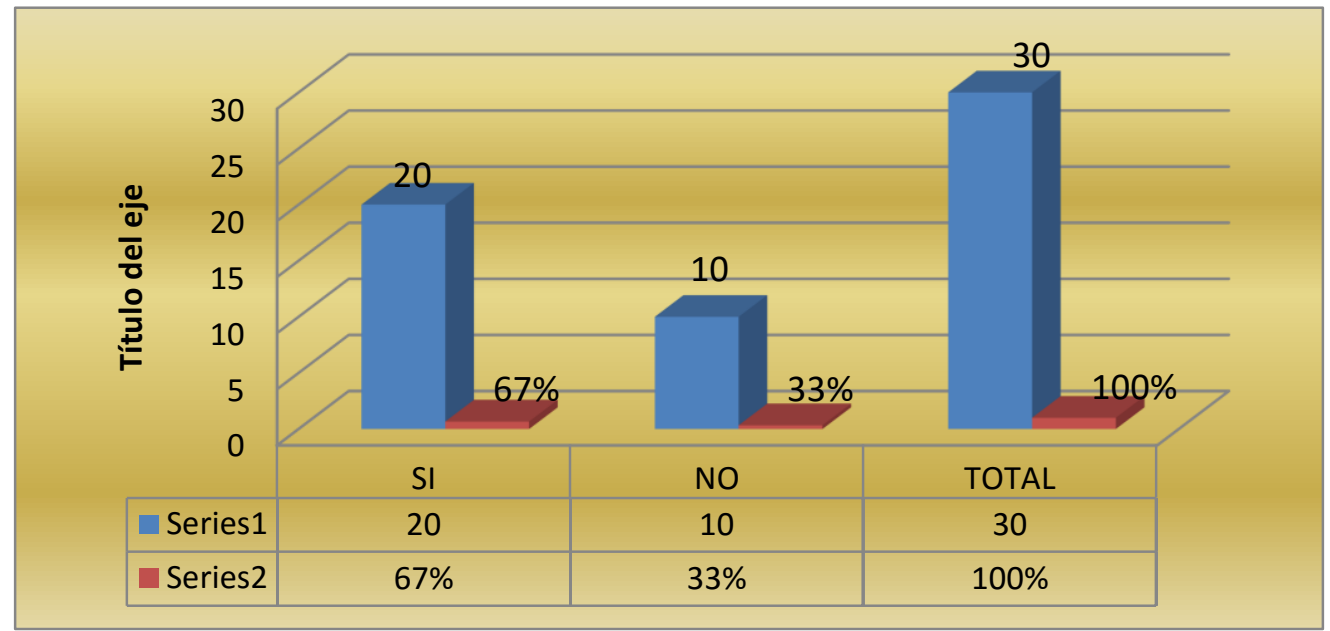

Gráfico 1. Estados Financieros conforme a NIIF.

Fuente: Milagro - encuesta realizada de Junio-Octubre del 2015.

Elaboración: propia - 2016.

En el objetivo específico, donde se formula "Determinar los factores de no aplicación de NIIF en las PYME del cantón Milagro", fue validada la hipótesis con la pregunta 4 ¿Qué dificultades se le ha presentado para la aplicación de las NIIF para las PYME?, considerando factores como la parte económica o financiera, conocimiento y resistencia al cambio o control interno eficiente, observar Gráfico 2.

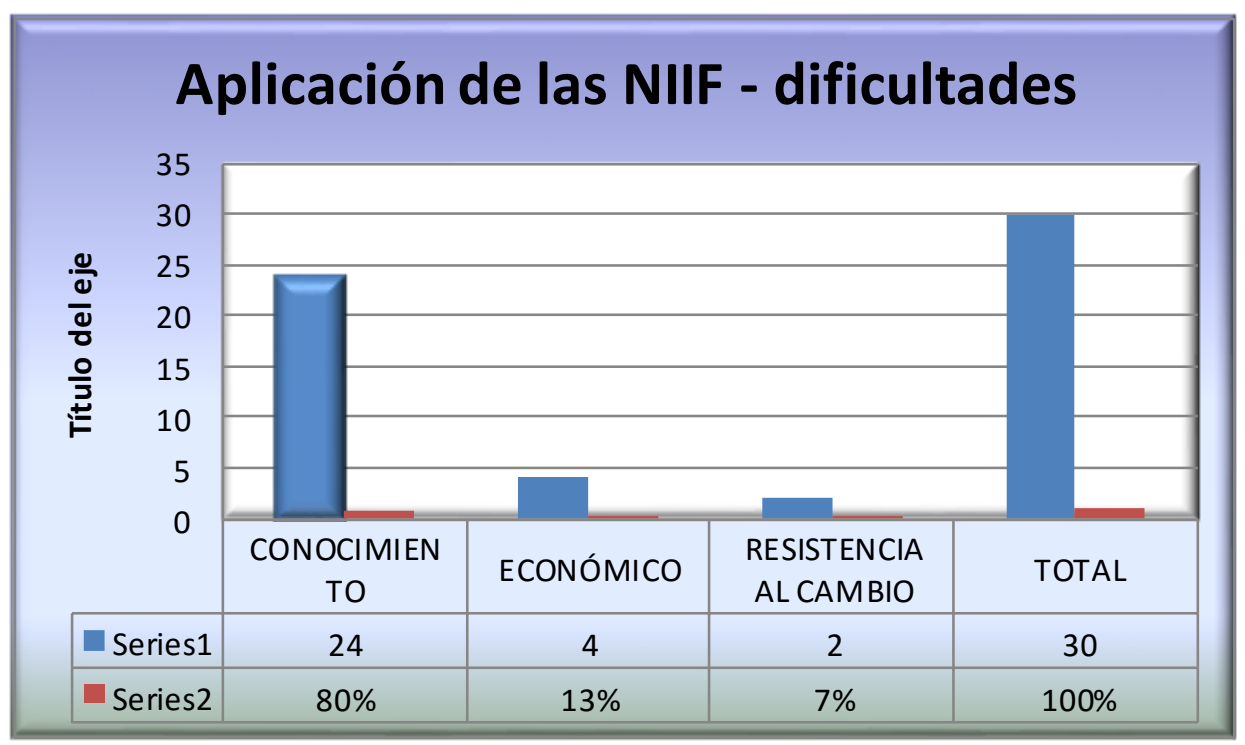

Gráfico 2. Factores que afectan a la aplicación de NIIF en las PYME.

Fuente: Milagro - encuesta realizada de Junio-Octubre del 2015.

Elaboración: propia -2016. 
El mayor peso de dificultad se demostró en el factor del "conocimiento", el $80 \%$ de la población encuestada no tiene claro la aplicación en NIIF para PYME, sus contadores aun manejan flujos de efectivos y no se basan en la acumulación o devengo.

Otra pregunta de vital importancia y modificada por las apreciaciones de los expertos académicos fue ¿Sería de gran ayuda si contaran con una guía o manual para indicar como se debe aplicar las NIIF para las PYME?

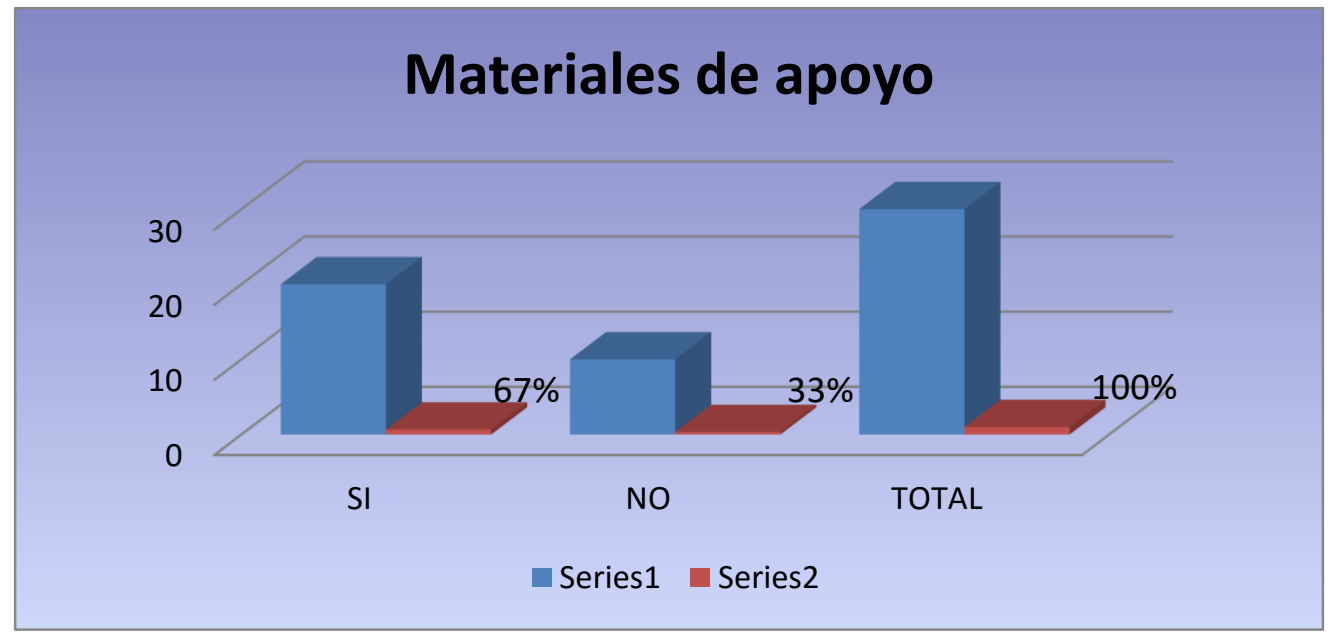

Gráfico 3. Material de apoyo para las PYME.

Fuente: Milagro - encuesta realizada de Junio-Octubre del 2015.

Elaboración: propia -2016.

En el Gráfico 3 se corrobora que 10 PYME, consideradas en la tabla 3, no requieren de información de apoyo, esto es sostenible en virtud que ellas son personas jurídicas o sociedades y cuentan con departamentos exclusivos en áreas financieras y contables, sin embargo el (67\%) restante, que equivale a las 20 PYME, si requieren de capacitación e instructivos que les permita medir y realizar sus estados financieros, conforme a las normas vigentes de Contabilidad.

\section{CONCLUSIONES}

Existe, dentro de las PYMES del cantón Milagro que comercializan electrodomésticos y por parte de los funcionarios y empleados involucrados en la elaboración de los Estados Financieros, un alto grado de desconocimiento conceptuales y prácticos sobre la aplicación de las Normas Internacionales de Información Financiera.

El 100\% de las PYMES dedicadas a la comercialización de electrodomésticos en el cantón Milagro, el 33\% están establecidas legalmente como persona jurídica, mientras que el $67 \%$ son personas naturales, obligadas a llevar Contabilidad. Las mismas que no entregan información a la Superintendencia de Compañías ni aplican NIIF. 
Que la dificultad que presentaron las PYMES durante el proceso de aplicación de NIIF se da por dos causas que son: el descuido por parte de la gerencia y la falta de capacitación al personal involucrado en NIIF, que a futuro afectarán a la rentabilidad de las PYMES. (Vargas \& Ramírez, 2016).

De acuerdo a las conclusiones establecidas se recomienda:

A los directivos de las PYMES, que organicen un plan de capacitación en la aplicación de NIIF, para el personal que intervienen en el proceso de elaboración de Estados Financieros.

Se sugiere la realización de estrategias que incluya a diversas PYMES, no solo en comercialización de electrodomésticos, sino servicios e industrialización. También resultaría interesante extender la investigación para medir resultados finales tanto en rentabilidad como la calidad del servicio que ofrecen los empleados, y el grado de conocimiento de los equipos de contadores que en ellos laboran (Audina \& Benavides, 2007).

Que a las personas Naturales obligadas a llevar Contabilidad se les capacite también en la aplicación de las NIIF, para la elaboración de sus Estados Financieros, ya que la competitividad obliga mantenerse actualizado.

\section{REFERENCIAS BIBLIOGRÁFICAS}

Audina, J. y Benavides, C. (2007). Ciencia, tecnología y desarrollo. Ministerio de Asuntos Exteriores y de Cooperación, Agencia Española de Cooperación Internacional. Recuperado de: < http://www.cce.co.cu/pdf/05-CienciaTecnologia.pdf/>.

Cano, A. (2010). Algunas experiencias exitosas en Latinoamérica relacionadas con la estandarización contable. Revista Facultad de Ciencias Económicas: Investigación y Reflexión, 18(2), 207-226. Recuperado de:

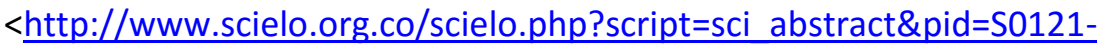
$\underline{68052010000200013 />\text {. }}$

Constitucional, T. (2008). Constitución de la República del Ecuador. Quito-Ecuador: Registro Oficial, 449, 20-10.

Compañías de, S. (2012). Cronograma de aplicación de NIIF en Ecuador. [en línea] Recuperado de:<https://es.scribd.com/doc/13333613/Cronograma-de-aplicacionde-NIIF-en-Ecuador/>

COMPAÑÍAS de, S. U. P. E. R. I. N. T. E. N. D. E. N. T. E. (2010). 1.-SUPERINTENDENCIA DE COMPAÑÍAS. Boletín №, 387(8).

Contabilidad de, C. D. N. I. (2009). NIIF para las PYMES. Norma Internacional de Información Financiera (NIIF) para Pequeñas y Medianas Entidades (PYMES). 
Fernández, M. (2010). Estudio del desarrollo productivo y socioeconómico de las pequeñas, medianas y microempresa del Cantón Milagro y cantones aledaños, y análisis de factibilidad para Interacción Institucional de Fortalecimiento" Dpto. de Investigación de la UNEMI, 2010, Milagro - Ecuador página 34-35.

Fosado, R. (2007). Información financiera en las PYMES. Revista del Centro de Investigación de la Universidad la Salle, 7(27), 67-75. Recuperado de: $<$ http://s3.amazonaws.com/academia.edu.documents/33721683/Informacion Fina nciera en las Pymes.pdf?AWSAccessKeyld=AKIAIWOWYYGZ2Y53UL3A\&Expires=14 86534194\&Signature=Oy749VKqJxHyHd684py|\%2B0jFq0w\%3D\&response-contentdisposition=inline\%3B\%20filename\%3DRedalyc.Informacion financiera en las PY.p df/>.

Gardey, G., Jorge, M., Fernández, A., \& Madueño, J. (2009). Influencia de la propiedad familiar sobre la eficiencia de las pymes: un análisis de diferencias basado en un concepto multicriterio de rentabilidad. Revista Internacional de la Pequeña y Mediana Empresa, 1(2), 42-63. Recuperado de: $<$ http://www.revistainternacionalpyme.org/volumenes/volumenuno/vol1num2/arti culos $/$ revista completa2. pdf\#page $=50 />$.

González, F. , Lacoba, S., Mera, A., \& Loureiro, S. M. C. (2015). Determinantes de la intención de uso de Facebook en el proceso de decisión de compra. Investigaciones Europeas de Dirección y Economía de la Empresa, 21(1), 26-34. DOI: $<$ http://dx.doi.org/10.1016/j.iedee.2014.05.001/>.

González, D. (2006). Regímenes especiales de tributación para pequeños contribuyentes en América Latina. Inter-American Development Bank. Recuperado de: $<$ http://s3.amazonaws.com/academia.edu.documents/33994149/IR01 Completa.pdf?AWSAccessKeyId=AKIAIWOWYYGZ2Y53UL3A\&Expires $=1486534650$

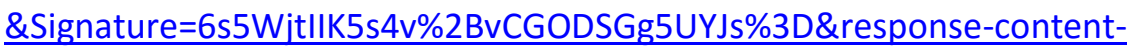
disposition=inline\%3B\%20filename\%3DRevista IR 01 Instituto AFIP ARGENTINA c .pdf\#page $=48 />$.

Casero, J. C. D., Mogollón, R. H., \& Urbano, D. (2005). Teoría económica institucional y creación de empresas. Investigaciones europeas de dirección y economía de la empresa, 11(3), 209-230. Recuperado de: <http://www.aedemvirtual.com/archivos/iedee/indiceiedee.htm/>.

Justiniano, M. F. (2016). El problema del crecimiento económico en la Europa moderna: del eurocentrismo al asiacentrismo y policentrismo. Revista tiempo\&economía, 3(1), 1937. $<\underline{\text { https://revistas.utadeo.edu.co/index.php/TyE/article/view/1093/>. }}$.

Lozares Colina, C. (2003). Valores, campos y capitales sociales. Redes: revista hispana para el análisis de redes sociales, 4 . Recuperado de: $<$ https://ddd.uab.cat/pub/redes/15790185v4/vol4 2.htm/>.

NIIF, Fundación IFRS (2009) "Material de formación sobre la NIIF para las PYMES" publicado por el Consejo de Normas Internacionales de Contabilidad, IASB Toronto. Recuperado de: $<$ http://www.ifrs.org/IFRS-for- 
SMEs/Documents/Spanish\%20IFRS\%20for\%20SMEs\%20Modules/Updated\%20S panish\%20IFRS\%20for\%20SMES\%202014/9 Consolidated\%20 Separate\%20Fina ncial\%20Statements 2013.pdf/>.

Marchán Rumbea, K. R. (2009). Análisis de competencias y habilidades para que un usuario utilice herramientas de inteligencia de negocios en las pymes del Ecuador. (Trabajo de fin de master inédito). Escuela Superior Politécnica del Litoral, Guayaquil, Ecuador.

Medina López, J. A. (2013). Guía de seguridad informática para la pymes de la ciudad de Pelileo.

Ontañeda, C (2008) Las empresas familiares se alían, Diario el Universo - Economía lunes 14 de abril del 2008.2 Recuperado <http://www.eluniverso.com/2008/04/14/0001/9/451B02D665C34712A17A1FFCA8 74FD53.html/>.

Oria, I. Z. (1997). La justificación y los efectos económicos del Impuesto de Sociedades. Ekonomiaz: Revista vasca de economía, (38), 122-145. Recuperado de: $<$ https://dialnet.unirioja.es/servlet/articulo?codigo=274247/>.

Pincay, D, \& Sánchez, J (2013) "Régimen Impositivo Simplificado Ecuatoriano en el Cantón Milagro: su crecimiento y evolución". Revista PODIUM Junio-2013 N.23, Guayaquil, Ecuador.

Rodrigo, J. B. (2006). La investigación en la Empresa Familiar: Un debate sobre la existencia de un campo independiente. Investigaciones europeas de dirección y economía de la empresa, 12(1), 33-54. Recuperado de: <http://www.aedemvirtual.com/archivos/iedee/indiceiedee.htm/>.

Rodríguez Valencia, J. (2000). Cómo aplicar la planeación estratégica en la pequeña y mediana empresa. Editorial Ecapsa-Thompson Learning.

Rodríguez, Carlos Eduardo (2014) La tributación de las Pymes en el Cantón Milagro. (Trabajo fin de master inédito) Universidad de Guayaquil, pág. 09 a la 27, Guayaquil, Ecuador.

Sánchez, J., \& Pincay, D. (2013). La Contabilidad Pública en América Latina y el Devengo en Ecuador. Analítika: revista de análisis estadístico, (6), 19-32. Recuperado de: $<$ http://www.ecuadorencifras.gob.ec/documentos/webinec/Revistas/Analitika/volum-multimedia/ANAlitica6/index.htm|\#/18/>.

Stumpo, G. (2004). Pequeñas y medianas empresas y eficiencia colectiva: estudios de caso en América Latina. Siglo XXI.

Tasigchana Aguilar, L (2015). Estudio para la aplicación de las Normas internacionales de Información Financiera (NIIF) en las Pymes en la toma de decisiones que están dedicadas a la comercialización de Electrodomésticos en el cantón Milagro provincia del Guayas. (Trabajo fin de master inédito) Universidad Estatal de Milagro, Milagro, Ecuador. 
Tobar Pesántez, L. (2013). Competitividad de las Microempresas en Cuenca, Ecuador (Competitiveness of Micro-Enterprises in Cuenca, Ecuador). Revista Internacional Administración \& Finanzas, 6(7), 121-129.

Urbano, D., Casero, J. C. D., \& Mogollón, R. H. (2007). La teoría económica institucional: el enfoque de North en el ámbito de la creación de empresas. In Decisiones basadas en el conocimiento y en el papel social de la empresa: XX Congreso anual de AEDEM ( $\mathrm{p}$. 35). Asociación Española de Dirección y Economía de la Empresa (AEDEM).

Vásquez, N. (2014). Estado actual de Latinoamérica en relación a su proceso de adopción de las NIIF. Contabilidad y auditoría, 19(38), 51-103 Recuperado de: <http://www.ojs.econ.uba.ar/ojs/index.php/Contyaudit/article/view/562/1034/>.

Vargas-Hernández, J. G., \& Ramírez, M. L. B. (2016). Estructura empresarial y competitividad en México. 3C Empresa: investigación y pensamiento crítico , 5(3), 26-53. DOI: <http://dx.doi.org/10.17993/3cemp.2016.050327.26-53/>.

Velasco, C. A. B., Parra, V. F. G., \& García, C. Q. (2011). Evolución de la literatura sobre empresa familiar como disciplina científica. Cuadernos de Economía y Dirección de la Empresa, 14(2), 78-90. DOI: <http://dx.doi.org/10.1016/i.cede.2011.02.004/>. 